\title{
Dynamic Functional Residual Capacity can be estimated using a Stress Strain Approach
}

\author{
A. Sundaresan ${ }^{1}$, J. G. Chase ${ }^{1}$, C. E. Hann $^{1}$ and G. M. Shaw ${ }^{2}$ \\ ${ }^{1}$ Department of Mechanical Engineering, University of Canterbury, Christchurch, \\ New Zealand \\ ${ }^{2}$ Department of Intensive Care, Christchurch Hospital, Christchurch, New Zealand
}

\section{Abstract}

\section{Background}

Acute Respiratory Distress Syndrome (ARDS) results in collapse of alveolar units and loss of lung volume at the end of expiration. Mechanical ventilation is used to treat patients with ARDS or Acute Lung Injury (ALI), with the end objective being to increase the dynamic functional residual capacity (dFRC), and thus increasing overall functional residual capacity (FRC). Simple methods to estimate dFRC at a given positive end expiratory pressure (PEEP) level in patients with ARDS/ ALI currently do not exist. Current viable methods are time consuming and relatively invasive.

\section{Methods}

Previous studies have found a constant linear relationship between the global stress and strain in the lung independent of lung condition. This study utilizes the constant stress strain ratio and an individual patient's volume responsiveness to PEEP to estimate dFRC at any level of PEEP. The estimation model identifies two global parameters to estimate a patient specific $\mathrm{dFRC}, \beta$ and $\mathrm{m} \beta$. The parameter $\beta$ captures physiological parameters of FRC, lung and respiratory elastance and varies depending on the PEEP level used, and $\mathrm{m} \beta$ is the gradient of $\beta$ vs PEEP. 


\section{Results}

dFRC was estimated at different PEEP values and compared to the measured dFRC using retrospective data from 12 different patients with different levels of lung injury. The median percentage error is 18\% (IQR: 6,49) for PEEP = $5 \mathrm{~cm}$ H2O, 10\% (IQR: 9,18) for PEEP = 7 cm H2O, 28\% (IQR: 12,33) for PEEP = 10 cm H2O, 3\% (IQR: 2,10) for PEEP = $12 \mathrm{~cm} \mathrm{H2O}$ and 10\% (IQR: 9,11) for PEEP = $15 \mathrm{~cm} \mathrm{H2O}$. The results were further validated using a cross correlation $(\mathrm{N}=100,000)$. Linear regression between the estimated and measured dFRC with a median R2 of 0.948 (IQR: $0.915,0.968 ; 90 \%$ CI: $0.814,0.984$ ) over the $\mathrm{N}=100,000$ cross validation tests.

\section{Conclusions}

The results suggest that a model based approach to estimating dFRC may be viable in a clinical scenario without any interruption to ventilation and can thus provide an alternative to measuring $\mathrm{dFRC}$ by disconnecting the patient from the ventilator. The overall results provide a means of estimating the dFRC at any level of PEEP with clinically reasonable accuracy for evaluating the impact of changes in PEEP or other mechanical ventilation settings. 


\section{Introduction}

Patients admitted to the intensive care unit (ICU) with acute lung injury (ALI) or its more severe form, acute respiratory distress syndrome (ARDS) [1-3] have mortality rates ranging from $20 \%$ to $70 \%$ [4]. ALI or ARDS occurs when the lung is inflamed and fills with fluid and cellular infiltrate causing a loss of functional lung units. This series of events results in a stiffer and smaller lung - the so called "baby lung" [5]. Currently, there are no specific treatments for ALI/ ARDS. Management is essentially supportive with an emphasis on minimising harm to the lungs, which results from suboptimal mechanical ventilation [6]. Typically, the severity of ARDS is measured as the ratio of the arterial partial pressure of oxygen divided by the fraction of inspired oxygen $\left(\mathrm{PiO}_{2} / \mathrm{FiO}_{2}\right.$ ratio). $\mathrm{A} \mathrm{PiO}_{2} / \mathrm{FiO}_{2}$ value less than 300 implies the patient has ALI, while anything lower than 200 is characterised by ARDS [7].

Mechanical ventilation is the primary treatment for ARDS and aids recovery by reducing or completely taking over the work of breathing. A positive end expiratory pressure (PEEP) is used to maintain a partially inflated lung at the end of expiration and to maintain recruitment during subsequent breathing cycles [8-11]. The optimal level of PEEP has been widely studied and debated with no conclusive results [8].

Functional Residual Capacity (FRC) represents the pulmonary gas volume of the lung at end expiration at atmospheric pressure. The objective of mechanical ventilation is to maximise gas exchange, while minimizing any additional lung injury due to over distension by using PEEP to increase FRC through the resulting alveolar recruitment. PEEP can be used to increase FRC, however there is a risk of overstretching healthy lung units in the process [12]. 
Currently, there are few methods of measuring FRC at the bedside. Gas washout/ washin techniques are one type of method [13], but are not readily available on most ventilators. A limitation of this type of measurement is that only an absolute value of FRC is measured. Hence, no information on the potential for new recruited lung volume is given, which is critical in setting PEEP to gain maximum recruitment with minimum added injury.

Figure 1 shows a schematic of the lung. When FRC is measured using a washout/ washin technique, it cannot be determined how many potential recruitable units are available. Therefore, as shown in the schematic, a lung with an FRC of 1.4 litres could be a result of a lung with 1.4 litres of fully recruited healthy lung units or 1.0 litres of recruited lung plus an additional amount of lung which has been recruited due to additional PEEP.

FRC can also be measured by using computed tomography (CT) scans [14]. Timed at the end of expiration, the gas volume can be assessed at each slice and summed across all the slices in the lung to evaluate true lung FRC. However, this type of measurement is unrealistic for regular use or monitoring in the ICU.

Although specialised ventilators may have FRC measurements (GE ventilators), most standard ventilators do not measure FRC, do not give the appropriate information required for optimal ventilator treatment, or require transportation of the patient (CT scanning). Thus, there is generally no practical bedside method that can be used to estimate recruited lung. Therefore, there is a motivation to be able to estimate the 
level of potential recruitment in the lung to help clinicians optimise mechanical ventilation treatment.

The ability to use standard ventilator data to estimate a dynamic FRC (dFRC), which includes recruited lung, would be a significant potential enhancement in ventilation management. Dynamic FRC is the level of additional lung volume that is achieved in the lung due to an additional level of applied PEEP and is shown schematically in Figure 2 [15]. Although dynamic FRC cannot by itself estimate the potential of lung recruitment, used with gas measurements it can provide the clinician with useful information on lung recruitability.

Thus, dynamic FRC value represents an aspect of the clinical endpoint in ventilation management, with the potential to be continuously tracked with changes in patient condition. This research develops a tool to estimate the level of additional pulmonary volume of collapsed alveolar units resulting from changes made to the applied inspiratory and expiratory airway pressures during mechanical ventilation. The method is based on identifying global parameters using a stress strain approach and estimating the level of additional lung volume due to PEEP. The potential for the model to be used in a clinical setting is explored based on the measurements required. 


\section{Method}

Retrospective clinical data from 12 patients was obtained with different levels of lung injury or ARDS from the study of Bersten et al [12]. Bersten et al reported 10 patients in this paper, however an additional two patients were not reported due to limited PV loops recorded. These data sets include PV loops for each patient at a minimum of 3 different PEEP values with a measured dFRC. PEEP was applied and held for 30 minutes before sampling was done. During the final 60 seconds, the data was then sampled at $100 \mathrm{~Hz}$. After approximately 40 seconds of tidal ventilation at PEEP, the ventilator is then set to zero end expiratory pressure (ZEEP), allowing the lung to deflate to FRC. Although this manouver does not measure FRC directly, it does capture FRC by measuring dynamic FRC, the extra volume due to PEEP which can lead to optimising lung recruitment.

Chiumello et al [16] studied the relationship between the global stress and strain during mechanical ventilation in ARDS patients. They defined the clinical equivalent of stress as the transpulmonary pressure $\left(\Delta P_{L}\right)$, while the strain was defined as the ratio of change in volume $(\Delta V)$ to the volume at the relaxed state of the lung or FRC. They also defined the specific lung elastance $\left(E_{L s p e c}\right)$ as the transpulmonary pressure at which FRC effectively doubles.

The relationship between stress and strain is defined [16]:

$$
\Delta P_{L}(\text { stress })=E_{\text {Lspec }} \times \frac{\Delta V}{F R C}
$$

where $\triangle V / F R C$ is the strain and $E_{L s p e c}$ is the specific lung elastance. The values of the specific lung elastance $E_{\text {Lspec }}$ in [16] are reported as $13.4 \pm 3.4$ for surgical control 
subjects, $12.6 \pm 3.0$ for medical control subjects, $14.4 \pm 3.6$ for ALI subgroup and $13.6 \pm 4.1 \mathrm{~cm} \mathrm{H}_{2} \mathrm{O}$ for the ARDS subgroup. This indicates that $E_{L s p e c}$ does not vary significantly within different groups and has a tight range of values.

The general relationship between the change in plateau airway pressure, $\Delta P_{a w}$, when the airflow is zero and the corresponding transpulmonary pressure is [16]:

$$
\begin{gathered}
\Delta P_{L}(\text { stress })=\Delta P_{a w} \times \alpha \\
\alpha=\frac{E_{L}}{E_{L}+E_{C W}}
\end{gathered}
$$

where $\alpha$ represents the static lung elastance and represents the ratio of the lung elastance $\left(E_{L}\right)$ to the chest wall elastance $\left(E_{C W}\right)$. It is critical to understand the importance of $\alpha$ in mechanical ventilation therapy. When a given airway pressure is applied, part of the pressure is used to inflate the lungs, with the remaining used to inflate the chest wall. The aim of mechanical ventilation is to ventilate patients while simultaneously trying to minimise the stresses exhibited in the lungs. Figure 3 shows the effect of different elastance values for the lungs and chest wall. Although in both cases, the total elastance is the same, Figure 3(a), typical of an ARDS patient, would experience a higher lung stress than Figure 3(b) as the lung is stiffer due to the higher elastance. Thus, $\alpha$ gives an indication of the severity of the ARDS affected lung.

The values of $\alpha$ in [16] varied from $0.69 \pm 0.15$ for surgical control subjects, $0.74 \pm$ 0.16 for medical control subjects, $0.64 \pm 0.15$ for the ALI subgroup and $0.71 \pm 0.16$ for the ARDS subgroup, all of which showed that $\alpha$ remained relatively constant across all patients with little deviation. 
The equation of motion for airway pressure is defined as:

$$
\Delta P_{a w_{-} t o t}=V \times E+Q \times R+P 0
$$

where $\Delta P_{a w_{-} \text {tot }}$ represents the total airway pressure, $V$ is the lung volume, $E$ is the respiratory elastance, $Q$ is the airflow rate, $R$ is the respiratory resistance and $P 0$ is the offset pressure which is a combination of applied PEEP and intrinsic PEEP [18, 19]. The equation of motion describes the total airway pressure as a function of the resistive and elastic components of the respiratory system. As resistance and elastance increase, the equation shows the airway pressure increases in order to overcome the respiratory resistance and the increased elastic recoil of the respiratory system.

In the case of no flow, the resistive term is zero. In this case, the airway pressure is purely a function of the respiratory elastance and is known as the plateau airway pressure, as shown in Equation (5).

$$
\Delta P_{a w}=V \times E+P 0
$$

Typically, if the pressure is measured, it is done at the ventilator or near the mask. Hence, all the PEEP data in this study represent plateau airway pressures. Because transpulmonary pressure is not typically measured at the bedside, it is estimated using the PEEP. Thus, rather than using the transpulmonary pressure $\Delta P_{L}$, the airway pressure is used as an estimate based on Equation (2).

At the beginning of inspiration, when the airway pressure is equal to PEEP, there is a point of zero flow, when the airflow reverses between expiration and inspiration. At this point in time, the volume is measured as dynamic FRC. Because plateau airway pressure occurs during zero flow, this justifies the use of PEEP and the corresponding dynamic FRC as a substitute for plateau airway pressure and lung volume. 
Combining Equations (1) and (2) yields a formula for FRC involving the two easily measured quantities $\Delta V$ and $\Delta P_{a w}=\Delta P E E P$ assumed here:

$$
F R C=\frac{\Delta V}{\Delta P E E P} \times \frac{E_{\text {Lspec }}}{\alpha}
$$

Equation (6) represents the FRC as a function of the specific lung elastance and the volume responsiveness of the patient. The data provided by Bersten et al did not include any FRC measurements. However, it did include the extra lung volume due to a given level of additional PEEP, or dynamic FRC. These values are graphically represented in Figure 4, where FRC is shown as the baseline, and dynamic FRC is measured relative to an unknown FRC value.

Figure 4 shows the dynamic FRC for a low and a high PEEP setting. The dynamic FRC is composed of FRC and the additional volume due to PEEP. As PEEP increases from $\mathrm{PEEP}_{1}$ to $\mathrm{PEEP}_{2}$, there is an increase in dynamic FRC. In a recruitment manoeuvre, additional PEEP is applied to re-inflate collapsed alveoli. Once the additional PEEP is removed, some of the re-inflated alveoli remain open and hence dynamic FRC is increased. Therefore physiologically, the change in dynamic FRC represents the $\triangle \mathrm{FRC}$ plus the change in alveolar recruitment due to PEEP. In this model, the FRC is not known, and the effect on FRC due to the recruitment manoeuvre is also not known, and typically is quite intensive or difficult to measure in a typical clinical situation. 
The aim of this research was to model the increase in dFRC due to PEEP. Thus, we hypothesised that the dFRC follows similar mathematical form to Equation (6). The given PEEP and dFRC in Figure 4, correspond to $\Delta V=\triangle d F R C$ and $\triangle P_{a w}=\triangle P E E P$.

$$
F R C+d F R C=\frac{\Delta d F R C}{\triangle P E E P} \times \frac{E_{\text {Lspec }}}{\alpha}(1+x)
$$

Therefore, the dynamic FRC takes the form:

$$
d F R C=\frac{\Delta d F R C}{\triangle P E E P} \times \frac{E_{\text {Lspec }}}{\alpha} \times x
$$

where $x$ is a function of the PEEP level that dynamic FRC is to be estimated at.

Because $E_{L \text { spec }}$ and $\alpha$ are constant values, this suggests that these parameters can be lumped into a single parameter. Therefore an alternative model for estimating dFRC is developed:

$$
d F R C=\frac{\Delta d F R C}{\triangle P E E P} \times \beta
$$

where $\beta$ is a function of the PEEP level. It must be noted, the assumption that $\alpha$ remain constant only holds for the linear portion of the static PV curve.

Re-arranging Equation (6) to solve for $\triangle d F R C / \triangle P E E P$, and substituting into Equation (9), we can solve for $\beta$, where $\beta$ is:

$$
\beta=\frac{E_{\text {Lspec }}}{\alpha} \times \frac{d F R C}{F R C}
$$

The term $\triangle d F R C / \triangle P E E P$ indicates the volume responsiveness of the patient to the specified change in PEEP. Thus, for a given PEEP, we have hypothesised that $\beta$ can be assumed to be constant over all patients.

With PEEP increasing, the dynamic FRC also increases as more recruitment occurs as seen in Figure 4. Because $\beta$ is linearly related to dFRC as shown in Equation (10), $\beta$ is also a function of the PEEP. Thus, $\beta$ represents the potential for additional recruitment 
based the given level of PEEP and also accounts for the level of damage to the lung by taking into account the elastances of the chest wall and lung.

In the clinical setting, the dynamic FRC is a parameter that is not normally measured and it is this variable that the model estimates. Because dynamic FRC is a function of PEEP, and $\beta$ is a function of dynamic FRC, it is possible to combine parameters such that $\beta$ is a function of PEEP. This then provides a method to estimate dynamic FRC using known $\beta$ and PEEP.

Because FRC was not known for any patient, $\beta$ was analytically solved based on Equation (9) using the measured dFRC value from the data. Once $\beta$ values were calculated for each patient at each PEEP value, a median $\beta$ was then evaluated for each PEEP level. This median value was then approximated as a $\beta$ value for a given PEEP across the entire population. The dFRC was then estimated using this median $\beta$ value. The overall process is summarised in Figure 5.

The median and interquartile range non parametric statistics were chosen to be the summary statistics to report. Because of the limited number of data points available ( $\mathrm{N}=39$ PV loops to predict from), the distribution of errors was non-Gaussian. In such situations it is better to present the median which is a true representation of the central tendency and the $50^{\text {th }}$ percentile [20]. As the number of data points increases, the mean value would tend to converge to the median value if the distribution was Gaussian. 
The dFRC values that were estimated using the median $\beta$ value were compared with the actual dFRC values measured to measure the estimate error over the method and assumed population constant value of $\beta$.

The model based method was validated using a correlation test with the measured clinical dFRC. The data provided 40 different dFRC measurements at various PEEP levels. Thus, $80 \%$ of the data (32 data points) were randomly selected without replacement and the median $\beta$ value found for each PEEP along with the gradient of $\beta$ vs PEEP, or $\mathrm{m} \beta$.

Using these calculated parameters from $80 \%$ of the data, the dFRC was then estimated for the remaining $20 \%$ of the data. A linear regression was performed by comparing the estimated dFRC with the measured dFRC for the remaining $20 \%$ of the data (8 data points). This process was then repeated 100,000 times and summary statistics reported.

This repetition ensures a wide and reasonably exhaustive coverage of possible test (n $=8)$ and validation $(n=32)$ sets are examined. This approach eliminates or reduces the potential that random chance or specific patient data sets skewed the results. It thus serves to statistically validate the general modelling and analysis approach presented. 


\section{Results}

Table 1 shows the analytical solution of $\beta$ for each PEEP level over all 12 patients.

The median and IQR are shown. The dFRC for each PEEP value was then estimated using the median value of $\beta$ at that PEEP value.

Table 1- Exact and Median Values of $\beta$ for different PEEP for all patients

\begin{tabular}{|l|r|r|r|r|r|r|}
\hline PEEP [cm H $\mathbf{2}$ ] & $\mathbf{0}$ & $\mathbf{5}$ & $\mathbf{7}$ & $\mathbf{1 0}$ & $\mathbf{1 2}$ & $\mathbf{1 5}$ \\
\hline Patient & & & & & & \\
\hline $\mathbf{1}$ & & 4.67 & 7.14 & & 11.79 & \\
\hline $\mathbf{2}$ & 0.48 & 5.31 & & 10.48 & & \\
\hline $\mathbf{3}$ & & 6.10 & 7.96 & 11.09 & & \\
\hline $\mathbf{4}$ & & 2.96 & 5.25 & 8.00 & & \\
\hline $\mathbf{5}$ & & 4.96 & 6.75 & 9.12 & 12.23 & \\
\hline $\mathbf{6}$ & & 2.07 & 3.58 & 6.79 & 8.98 & \\
\hline $\mathbf{7}$ & & 4.49 & 6.51 & 9.50 & 11.49 & \\
\hline $\mathbf{8}$ & & 3.15 & 5.67 & & 10.28 & \\
\hline $\mathbf{9}$ & & & & 10.13 & 12.44 & 15.17 \\
\hline $\mathbf{1 0}$ & & 4.73 & 7.23 & 9.75 & 11.94 & \\
\hline $\mathbf{1 1}$ & & 3.85 & 5.95 & 8.86 & & 12.43 \\
\hline $\mathbf{1 2}$ & & 2.43 & & 6.88 & & 13.80 \\
\hline Median & 0.48 & 4.49 & 6.51 & 9.31 & 11.79 & \\
\hline IQR & N/A & {$[3.06,4.85]$} & {$[5.67,7.14]$} & {$[8.22,10.04]$} & {$[10.89,12.09]$} & {$[13.12,14.49]$} \\
\hline
\end{tabular}

Table 2 shows the percentage error between the clinically measured dFRC and the dFRC estimated with the model. Figure 6 shows the general trend of clinical dFRC vs predicted dFRC for a PEEP of $5 \mathrm{cmH}_{2} \mathrm{O}$ across all patients, yielding $\mathrm{R}^{2}=0.712(\mathrm{R}=$ 0.845). This relatively low linear trend is attributed to the limited number and range of data points at the one PEEP level. 
Table 2 - Percentage Error of actual dFRC and predicted dFRC for all patients at all PEEP levels

\begin{tabular}{|c|c|c|c|c|c|c|}
\hline PEEP [cm H $\left.{ }_{2} \mathrm{O}\right]$ & 0 & 5 & 7 & 10 & 12 & 15 \\
\hline \multicolumn{7}{|l|}{ Patient } \\
\hline 1 & & 3.93 & 8.86 & & 0.01 & \\
\hline 2 & 0.52 & 15.41 & & 11.19 & & \\
\hline 3 & & 26.44 & 18.19 & 16.02 & & \\
\hline 4 & & 51.55 & 24.06 & 16.40 & & \\
\hline 5 & & 9.50 & 3.54 & 2.13 & 3.62 & \\
\hline 6 & & 116.52 & 81.97 & 37.14 & 31.24 & \\
\hline 7 & & 0.02 & 0.00 & 2.00 & 2.58 & \\
\hline 8 & & 42.65 & 14.75 & & 14.70 & \\
\hline 9 & & & & 8.10 & 5.19 & 9.02 \\
\hline 10 & & 5.11 & 9.98 & 4.53 & 1.24 & \\
\hline 11 & & 16.69 & 9.42 & 5.07 & & \\
\hline 12 & & 84.95 & & 35.27 & & 11.04 \\
\hline Median & 0.52 & 16.69 & 9.98 & 9.64 & 3.62 & 10.03 \\
\hline IQR & $N / A$ & {$[7.31,47.10]$} & {$[8.86,18.19]$} & {$[4.66,16.30]$} & {$[1.91,9.94]$} & {$[9.52,10.54]$} \\
\hline
\end{tabular}

Figure 7, which shows the predicted dFRC versus the measured dFRC across all patients at all PEEP levels shows greater linearity over all PEEP values with $\mathrm{R}^{2}=$ $0.947(\mathrm{R}=0.973)$. The relatively lower $\mathrm{R}^{2}$ value in Figure 6 could thus also be attributed to the relatively low range of dFRC values as compared to Figure 7 . Hence, the linear trend in clinical vs predicted dFRC is sustained over all PEEP values and a very wide range of dFRC.

In order to show the range of errors for the predicted dFRC, Figure 8 shows a Bland Altman plot with a $90 \%$ confidence. Although a few points exist outside the interval, the majority of the points are within a $90 \%$ confidence interval of the mean indicating that there the maximum deviation from measured dFRC is around $200 \mathrm{ml}$.

The summary statistic and results in the cross validation test are shown in Figure 9 and Table 3. Figure 9 shows the median $\beta$ across all patients and PEEP levels and clearly shows a linear relationship with PEEP. 
Table 3 shows the statistical results for the $\mathrm{R}^{2}$ correlation coefficient between the estimated and actual dFRC. It also shows the value of $m \beta$ across all PEEP values as per Figure 9 and the $\beta$ for each PEEP level. As shown, a 90\% confidence interval still produces a very high correlation coefficient. The low variability in $m \beta$ and $\beta$ indicates the potential to label them as global parameters that can be used to describe any patient at this level of PEEP.

Table 3 - 90\% Median IQR and Confidence Interval of R2 correlation coefficient, $\mathrm{m} \beta$ and $\beta$ at every level of PEEP

\begin{tabular}{|l|c|c|c|}
\hline & Median & IQR & 90\% CI \\
\hline $\mathbf{R}^{2}$ Coefficient & 0.944 & {$[0.933,0.952]$} & {$[0.910,0.960]$} \\
\hline Beta Gradient $\mathbf{( m \boldsymbol { \beta } )}$ & 0.953 & {$[0.877,1.046]$} & {$[0.804,1.164]$} \\
\hline Beta at PEEP = $\mathbf{~ c m H 2 O}$ & 4.065 & {$[3.695,4.458]$} & {$[3.057,5.020]$} \\
\hline Beta at PEEP = 7 cmH2O & 6.257 & {$[5.823,6.680]$} & {$[5.122,7.233]$} \\
\hline Beta at PEEP = 10 cmH2O & 9.074 & {$[8.628,9.520]$} & {$[7.883,10.190]$} \\
\hline Beta at PEEP = 12 cmH2O & 11.371 & {$[10.873,11.887]$} & {$[10.235,12.230]$} \\
\hline Beta at PEEP = 15 cmH2O & 13.800 & {$[12.512,15.170]$} & {$[12.430,15.170]$} \\
\hline
\end{tabular}

Figure 9 showed a linear relationship between $\beta$ and PEEP. At a PEEP $=0 \mathrm{cmH}_{2} \mathrm{O}$, the dynamic FRC value should be zero, and hence the linear relationship between $\beta$ and PEEP is described as:

$$
\frac{\beta}{P E E P}=m \beta
$$




\section{Discussion}

\section{Stress Strain Relationship}

The ability of using lung stress and strain as a proxy produces a reasonable estimate to the dynamic FRC. For the clinical data set used, the study indicated that $\beta$ increased very linearly as a function of PEEP. Chiumello et al [16] indicated that the ratio of lung elastance to total respiratory elastance $(\alpha)$ varied between a value of 0.33 to 0.95 over various PEEP settings. Gattinoni et al [21] showed that the respiratory mechanics may vary in patients depending on whether the ARDS originated from pulmonary or extrapulmonary disease [21]. They concluded that, depending on the origin of ARDS, the total respiratory elastance could increase or decrease as a function of increasing PEEP. However, no significant changes for $\beta$ occured in this study, as in [16], implying that $E_{L s p e c}$ and $\alpha$ are interdependent on each other.

In this analysis, because $\beta$ incorporates the dynamic FRC, FRC, $E_{L s p e c}$ and $\alpha$ according to Equation (9), then as the $\beta$ increases the value of dFRC also increases linearly. Hence, mathematically, $\beta$ is linearly related to dFRC, so that as $\beta$ increases, dynamic FRC also increases.

\section{Model Parameters}

The model uses 2 parameters to estimate the dynamic FRC for a given PEEP. The parameters are $\beta$ and $m \beta$, where $m \beta$ is the gradient of the line of $\beta$ vs PEEP, as shown in Figure 9. Cross correlation and validation showed very tight ranges around high values of $\mathrm{R}^{2}$. This result implies that $\beta$ and $\mathrm{m} \beta$ can be used as universal parameters across all PEEP levels to estimate the dFRC and its change for a given PEEP, as well 
as for any ARDS patient. However, this conclusion must be subject to validation in a larger clinical trial or with a larger equivalent clinical data set given the small patient numbers in this study.

\section{Clinical Implications}

The estimation of a dynamic FRC will be highly useful in the clinical setting. Although dFRC by itself only gives information on the additional lung volume due to PEEP changes, it can be used in conjunction with gas measurements to help model the recruitment potential of the lung. In particular, it can be combined with existing models of gas exchange [22] to create a fully modelled based approach to estimating lung recruitment. Clinicians can thus predict the amount of extra recruitment due to an increase in PEEP before applying it to a patient. However, to evaluate the dFRC according to Equation (9), a series of $\triangle \mathrm{PEEP}$ or incremental pressure holds must be performed to evaluate $\triangle d F R C / \triangle P E E P$. Given only three such results, it is possible to estimate the volume responsiveness of the patient and apply Equation (9).

Another implication of this model is as a means to estimate FRC. According to Equation (10), $\beta$ is related to the value of $\alpha, E_{L s p e c}$ and dFRC at a given PEEP. Assuming a constant value of $\alpha$ and $E_{\text {Lspec }}$ and using the predicted dFRC in this study, Equation (10) can be used to find an average value of FRC. Using an average value of $E_{\text {Lspec }}=13.6 \mathrm{~cm} \mathrm{H}_{2} \mathrm{O}$ and $\alpha=0.7$, the predicted FRC ARDS patients was $875 \mathrm{ml}$, which is within the values reported by Chiumello et al [16] of $1013 \pm 593 \mathrm{ml}$. This result further validates the model and methods presented. 
However, in reality, the value of $\alpha$ and $E_{\text {Lspec }}$ varies and FRC can thus also take on a range of values. This range of FRC values can then give an indication of the level of recruited lung without any mechanical ventilation treatment and, as a result, an indication of the total lung damage in the patient. Therefore, tracking this value daily, or more frequently, could potentially see increasing estimated mean FRC and thus provide an indicator on when to begin weaning a patient from mechanical ventilation.

\section{Specific Lung Elastance}

Chiumello defined the specific lung elastance as the transpulmonary pressure at which FRC doubles [16] and measured $E_{\text {Lspec }}$ using the supersyringe technique and inflating the lungs with an additional volume equal to FRC. In the model presented in this study, when dFRC equals FRC, the volume of the lung has essentially doubled (total lung volume equalling FRC plus dFRC), and this results in a $\beta$ value of $19.3 \mathrm{cmH}_{2} \mathrm{O}$ (using average values for $\alpha=0.7, E_{\text {Lspec }}=13.6 \mathrm{cmH} 2 \mathrm{O}$ ). Using the median value for $m \beta$ shown in Table 3 and Equation (11), the PEEP which produces this $\beta$ value is calculated to be $18.4 \mathrm{cmH}_{2} \mathrm{O}$. According to Equation (10), this corresponds to an equivalent transpulmonary pressure of $12.9 \mathrm{cmH}_{2} \mathrm{O}$. In this study, the value of $E_{\text {Lspec }}$ is therefore evaluated to be $12.9 \mathrm{~cm} \mathrm{H}_{2} \mathrm{O}$ which falls within the limits found by [16].

Although this is not the exact value, the difference in specific lung elastance values could arise from the fact that different data sets were used. This study used data from Bersten [12] with patients who had severe ARDS. In Chiumello’s study, the specific lung elastance in ARDS patients had a range from 9.5 to $17.7 \mathrm{~cm} \mathrm{H2O}$. The value obtained in this study falls within the reported range for ARDS patients. Secondly, [12] reported $\mathrm{PiO}_{2} / \mathrm{FiO}_{2}$ values as low as 66 , however the ranges reported by [16] for 
ARDS patients had a minimum value of 83. This difference could also cause the specific lung elastance to be slightly lower, as the more significant the ARDS, the less applied pressure required for doubling the FRC.

\section{Limitations}

Although the method predicts dFRC, there are some severe limitations to the predictive capability. At lower PEEP the error for predicted dFRC was shown to be as high as $120 \%$ as shown on Table 2 . As PEEP increases, the percentage error drops but some patients exhibit a difference of about $200 \mathrm{ml}$ between measured and predicted dFRC. At lower PEEP, a difference of $200 \mathrm{ml}$ and can cause the predicted dFRC to be very different than true dFRC. Despite a high $r^{2}$ value shown on Figure 7 , the predicted value either over states or understate the amount of potential lung volume that is being recruited. In the case of the lower PEEP settings, a large prediction error therefore can limit the potential estimator method.

Another limitation with this model is the assumption $E_{\text {Lspec }}$ and $\alpha$ being constant. This assumption only holds during the linear portion of the static PV curve. The linear portion of the static PV curve is where most of the data points occur on any measured dynamic PV loop. Based on the work of Sundaresan et al [23], over $90 \%$ of the data points from measured PV loops occur in the static portion of the static PV curve and are the points primarily used in this analysis. The non-linear portions at PEEP and PIP are actually high flow regions and are thus do not account for or include the majority of the data. This is further highlighted by assuming that $\triangle d F R C / \triangle P E E P$, effectively the compliance, does not change as a function of PEEP. An estimate of the static PV curve was plotted using the points of zero flow from each of the PV loops and is 
shown in Figure 10. A linear line of best fit was plotted and the $\mathrm{R}^{2}$ coefficient was computed to show if the estimated static PV curve was linear or non linear showing there and in Table 4 that the linear assumptions and data points used hold almost exclusively.

Table 4 - Linearity of static PV curve for each patient.

\begin{tabular}{|c|c|c|c|}
\hline Patient & $\mathbf{R}^{\mathbf{2}}$ & Patient & $\mathbf{R}^{\mathbf{2}}$ \\
\hline $\mathbf{1}$ & 0.9977 & $\mathbf{7}$ & 1.0000 \\
\hline $\mathbf{2}$ & 0.9998 & $\mathbf{8}$ & 0.9971 \\
\hline $\mathbf{3}$ & 0.9995 & $\mathbf{9}$ & 0.9978 \\
\hline $\mathbf{4}$ & 0.9981 & $\mathbf{1 0}$ & 0.9972 \\
\hline $\mathbf{5}$ & 0.9885 & $\mathbf{1 1}$ & 0.9998 \\
\hline $\mathbf{6}$ & 0.9975 & $\mathbf{1 2}$ & 0.9980 \\
\hline
\end{tabular}

Finally, the data used in this study is considered to be idealised. In each case, the PEEP is held for 30 minutes before any sampling was done. In a clinical setting, holding two or three PEEP settings for 30 minutes to measure $\triangle d F R C / \triangle P E E P$ is not practical. Thus, the model may be limited in the clinic as in reality it would be difficult to hold for 30 minutes. Therefore, in order for clinical application, the $\triangle d F R C / \triangle P E E P$ would have to be estimated without the 30 minute hold on PEEP. 


\section{Conclusions}

The research presented provides an easy to use tool that can be implemented in the intensive care unit. By monitoring or tracking changes in patient respiratory mechanics, the clinician is able to evaluate the potential of recruitable lung in the patient. This in turn can help understand what the optimal level of PEEP that is required during mechanical ventilation. The research method provides an easy to use tool without any special ventilator or the use of time consuming methods such as CT scans. 


\section{Competing Interests}

No Competing Interests

\section{Author Contribution}

AS, JGC \& CEH developed the model and performed statistical analysis on the data.

GMS helped with the data acquisition and validated statistical analysis

RWW helped with the validation of statistical methods

All authors read and approved the final manuscript. 


\section{References}

1. Esteban A, Anzueto A, Frutos F, et al., Characteristics and outcomes in adult patients receiving mechanical ventilation: a 28-day international study. Jama 2002; 287; 3; 345-55.

2. Hughes M, Grant I, MacKirdy F, et al., Incidence and Mortality after Acute Respiratory Failure and Acute Respiratory Distress Syndrome in Sweeden, Denmark and Iceland. Am J Respir Crit Care Med 2000; 162; 1; 332-3.

3. Walsh TS, Dodds S, McArdle F, Evaluation of simple criteria to predict successful weaning from mechanical ventilation in intensive care patients. Br J Anaesth 2004; 92; 6; 793-9.

4. Bersten AD, Edibam C, Hunt T, Moran J, Incidence and Mortality of Acute Lung Injury and the Acute Respiratory Distress Syndrome in Three Australian States. Am $J$ Respir Crit Care Med 2002; 165; 4; 443-8.

5. Gattinoni L, Pesenti A, The concept of "baby lung". Intensive Care Medicine 2005; 31; 6; 776 - 84.

6. Ware LB, Matthay MA, The Acute Respiratory Distress Syndrome. N Engl J Med 2000; 342; 18; 1334-49.

7. Bernard G, Artigas A, Brigham K, et al., The American-European Consensus Conference on ARDS. Definitions, mechanisms, relevant outcomes, and clinical trial coordination. Am J Respir Crit Care Med 1994; 149; 3; 818-24.

8. Amato MBP, Barbas CSV, Medeiros DM, et al., Effect of a Protective-Ventilation Strategy on Mortality in the Acute Respiratory Distress Syndrome. $N$ Engl J Med 1998; 338; 6; 347-54.

9. Network. TARDS, Ventilation with lower tidal volumes as compared with traditional tidal volumes for acute lung injury and the acute respiratory distress syndrome. N Engl J Med 2000; 342; 18; 1301-8.

10. McCann UG, Schiller HJ, Carney DE, et al., Visual validation of the mechanical stabilizing effects of positive end-expiratory pressure at the alveolar level. J Surg Res 2001; 99; 2; 335-42.

11. Halter JM, Steinberg JM, Schiller HJ, et al., Positive End-Expiratory Pressure after a Recruitment Maneuver Prevents Both Alveolar Collapse and Recruitment/Derecruitment. Am J Respir Crit Care Med 2003; 167; 12; 1620-6.

12. Bersten $A D$, Measurement of overinflation by multiple linear regression analysis in patients with acute lung injury. Eur Respir J 1998; 12; 3; 526-32. 
13. Heinze H, Schaaf B, Grefer J, et al., The Accuracy of the Oxygen Washout Technique for Functional Residual Capacity Assessment During Spontaneous Breathing. Anesth Analg 2007; 104; 3; 598-604.

14. Malbouisson LM, Muller JC, Constantin JM, et al., Computed Tomography Assessment of Positive End-expiratory Pressure-induced Alveolar Recruitment in Patients with Acute Respiratory Distress Syndrome. Am J Respir Crit Care Med 2001; 163; 6; 1444-50.

15. Amir K, Thomas GK, Robert d, Manuel D, Comparison of dynamic and passive measurements of respiratory mechanics in ventilated newborn infants. Pediatric Pulmonology 1995; 20; 4; 258-64.

16. Chiumello D, Carlesso E, Cadringher P, et al., Lung Stress and Strain during Mechanical Ventilation for Acute Respiratory Distress Syndrome. Am J Respir Crit Care Med 2008; 178; 4; 346-55.

17. Gattinoni L, Chiumello D, Carlesso E, Valenza F, Bench-to-bedside review: Chest wall elastance in acute lung injury/acute respiratory distress syndrome patients. Critical Care 2004; 8; 5; 350 - 5.

18. Carvalho A, Jandre F, Pino A, et al., Positive end-expiratory pressure at minimal respiratory elastance represents the best compromise between mechanical stress and lung aeration in oleic acid induced lung injury. Critical Care 2007; 11; 4; R86.

19. Eberhard L, Guttmann J, Wolff G, et al., Intrinsic PEEP monitored in the ventilated ARDS patient with a mathematical method. J Appl Physiol 1992; 73; 2; 479-85.

20. Micceri T, The unicorn, the normal curve, and other improbable creatures. Psychological Bulletin 1989; 105; 1; 156-66.

21. Gattinoni L, Pelosi P, Suter PM, et al., Acute Respiratory Distress Syndrome Caused by Pulmonary and Extrapulmonary Disease . Different Syndromes? Am J Respir Crit Care Med 1998; 158; 1; 3-11.

22. Rees SE, Kjærgaard S, Thorgaard P, et al., The Automatic Lung Parameter Estimator (ALPE) System: Non-Invasive Estimation of Pulmonary Gas Exchange Parameters in 10-15 Minutes. Journal of Clinical Monitoring and Computing 2002; $17 ; 1 ; 43-52$.

23. Sundaresan A, Yuta T, Geoffrey Chase J, et al., A minimal model of lung mechanics and model-based markers for optimizing ventilator treatment in ARDS patients. Computer Methods and Programs in Biomedicine 2009; 95; 2; 166-80. 